such a gravity anomaly is more than an order of magnitude greater than any actually measured. And as a result, Schubert and Turcotte conclude that the physical model is wrongthat is, that the balancing flow at depth is not limited to the asthenosphere. The balancing flow must be deeper in the mantle, possibly even below $700 \mathrm{~km}$ "and possibly throughout the entire mantle". The upper mantle immediately below the lithosphere must then be moving in the same, rather than opposite, direction as the lithosphere; and "it is likely that the dominant deformation mechanism is dislocation glide". In short, earth scientists are back where they were in the early 1960s.

\section{RIBOSOMAL RNA}

\section{Maturation Pathways}

from our Cell Biology Correspondent If the contents of recent issues of the Journal of Molecular Biology are anything to judge by over the past two or three years, more than a few molecular biologists in search of a quiet but honest way of earning their living have been attracted to the question of the biosynthesis of ribosomal RNAs in eukaryotes. Quasi-repetitive research, to use the journal's own description, it may be, but much current research does not fall in that bracket and those molecular biologists who have pursued the mechanism of ribosomal RNA synthesis can at least point to a large corpus of sound information as a result of their efforts. Indeed, eukaryotic ribosomal RNAs and their precursors are almost as well characterized as their bacterial counterparts.

Maden and his associates, for example (Nature New Biology, 237, 5 ; 1972 ), have by fingerprint analysis unambiguously confirmed the maturation pathway of HeLa cell ribosomal RNAs proposed by Penman and his associates and others from kinetic studies. And Udem and Warner (J. Mol. Biol., 65, 227 ; 1972), by measuring the kinetics of labelling of the short lived $35 \mathrm{~S}, 27 \mathrm{~S}$ and $20 \mathrm{~S}$ ribosomal RNA species as well as the stable, mature $25 \mathrm{~S}$ and $18 \mathrm{~S}$ species in spheroplasts of the yeast Saccharomyces cerevisiae, have been able to draw up a maturation pathway for this species. Udem and Warner conclude that the product of transcription of the ribosomal RNA genes is a single $35 \mathrm{~S}$ polynucleotide chain with a molecular weight of about $2.5 \times 10^{6}$ which on conservative cleavage yields one 27S RNA (molecular weight $1.6 \times 10^{6}$ ) and one $20 \mathrm{~S}$ RNA (molecular weight $0.8 \times 10^{6}$ ). These molecules are in turn cleaved, with the loss of about 20 per cent of the primary product of transcription, to yield the mature $25 \mathrm{~S}$ and $18 \mathrm{~S}$ species

by a terminal processing step, which is sensitive to inhibition by cycloheximide and is, presumably, dependent on continuous protein synthesis. The $5.8 \mathrm{~S}$ RNA, which is associated with the mature 25S ribosomal RNA and from which it can be released by denaturing agents, is apparently produced at the final cleavage step from the $27 \mathrm{~S}$ precursor RNA. Ribosomal 5S RNA, by contrast, seems to be the product of independent transcription of a separate gene.

As Udem and Warner point out, these data not only support the idea that in all eukaryotes the pathway of biosynthesis of ribosomal RNAs is essentially similar but also draw attention to the advantages of $S$. cerevisiae over cultivated animal cells for the experimentalist. They emphasize this point by describing in a subsequent report (ibid., 243) the isolation of mutant strains of yeast in which ribosomal RNA biosynthesis is temperature sensitive. Temperature sensitive mutations in nine genes all apparently inhibit to varying extents (50-80 per cent) the transcription of the $35 \mathrm{~S}$ precursor RNA and the subsequent maturation of what little precursor is made. Moreover, in the three mutant strains which have been examined, the synthesis of ribosomal proteins is reduced by about 75 per cent. Because neither precursor RNAs nor defective ribosomal particles or subunits accumulate in any of these mutant strains, Warner and Udem confidently conclude that the whole process of ribosome biosynthesis in this yeast is stringently regulated by some feedback inhibition mechanism.

Stevens and Pachler (J. Mol. Biol., 66, 225 ; 1972) contend that the low mole-

\title{
Virus Infection and Cell Agglutination
}

WHEN cells are transformed either by RNA or by DNA tumour viruses or by chemical carcinogens their susceptibility to agglutination by plant lectins such as concanavalin $A$ or wheat germ agglutinin is greatly increased. Burger has suggested that the changes in the cell surface consequent upon transformation which are reflected by the enhanced agglutinability may be responsible for the failure of transformed cells to respond to the molecular signals which regulate cell division. But as Poste and Reeve confirm in Nature New Biology next Wednesday (May 24) an increased susceptibility to agglutination by these two lectins is by no means a specific property of transformed cells.

The precise nature of the changes which cause transformed cells to be more readily agglutinated by lectins is obscure but nobody seriously doubts that they involve components of the cell coat and because many lytic, non- cular weight (4-6S) RNA which has been found associated by hydrogen bonds with the mature RNA of the large ribosomal subunit of some plant and animals cells is a universal and diagnostic characteristic of eukaryotic cells. Such RNA has never been found with the large ribosomal RNA of prokaryotes but, as they report, an RNA with a molecular weight of about $4.3 \times 10^{4}$ is liberated by denaturing agents from the $26 S$ ribosomal RNA of the protozoan Acanthamoeba castellani, a lowly eukaryote. What is more surprising, on denaturation the $26 \mathrm{~S}$ RNA itself seems to dissociate into two molecules sedimenting at 16-18S, and Stevens and Pachler believe, therefore, that the $26 \mathrm{~S}$ ribosomal RNA in this species comprises three RNA species with molecular weights of $0.88 \times 10^{6}, 0.6 \times 10^{6}$ and $4.3 \times 10^{4}$, held together by hydrogen bonds.

The ribosomal RNAs of a prokaryote, the trachoma agent which is an obligate parasite of eukaryocytes, have also recently been investigated as Gutter and Becker report (ibid., 239). They have identified in infected FL cells exposed to emetine which inhibits cellular RNA synthesis a 17.2S precursor of the mature $16 \mathrm{~S}$ ribosomal RNA of trachoma agent ribosomes and suggest that the mature $23 \mathrm{~S}$ ribosomal RNA may also be matured from a larger precursor. The maturation of the 17.2S precursor, which is a slow process, seems to be blocked by chloramphenicol and the synthesis of the precursor and its maturation is inhibited by puromycin. In short, although the trachoma agent has its idiosyncrasies, the synthesis and maturation of its ribosomal RNAs clearly follow the bacterial pattern.

oncogenic viruses are known to cause changes at the cell surface Reeve and Poste decided to compare the susceptibility to agglutination of BHK cells and chick embryo cells before and after infection with Newcastle disease virus. At the same time, in parallel experiments, Reeve and Poste measured the thickness of the cell coat with an ellipsometer.

Poste and Reeve's results indicate, first, that infection with Newcastle disease virus renders cells susceptible to agglutination by both concanavalin $\mathrm{A}$ and wheat germ agglutinin and, second, that as the cells become more readily agglutinable so the thickness of the cell coat decreases. When, however, cells are infected with a strain of Newcastle disease virus which replicates without altering the cell coat material the cells do not become susceptible to agglutination, neither does the cell coat layer become thinner; the two changes may therefore be related. 